\title{
A Virtual Reality Environment Using Concepts of Serious Games and Gamification for the Treatment of Eating Disorders
}

\author{
Francielly Rodrigues ${ }^{1}$, Priscila Wilbert ${ }^{2}$, \\ José Carlos Tavares da Silva ${ }^{3}$, Jauvane C. de Oliveira ${ }^{1}$ \\ ${ }^{1}$ National Laboratory for Scientific Computing (LNCC) \\ Petrópolis, RJ, Brazil \\ ${ }^{2}$ Faculdade Arthur Sá Earp Neto - Petrópolis, RJ, Brazil \\ ${ }^{3}$ Department of Computer Science - Fundação Educacional Serra dos Órgaos \\ Teresópolis, RJ, Brazil \\ \{fmunique, jauvane\}@lncc.br ${ }^{1}$, priscilawilbertegmail.com ${ }^{2}$ \\ tavaresequatra.com.br ${ }^{3}$
}

\begin{abstract}
An eating disorder is a disturb characterized by an atypical eating behavior. The most common strategy for treatment is CBT, and its limitations have shown to be overcome using virtual reality approaches. Most VR environments created for this purpose reproduce tasks for treatment of body image disturbances and food avoidance. This work presents a new approach for eating disorders treatment using VR and concepts of serious games and gamification, which have been shown of promising potential in health context due to its advantages when compared to other approaches. Tests assessing the usability and sense of presence in the virtual environment were applied to a group of 10 participants, indicating that the environment is well suited for medical treatment.
\end{abstract}

\section{Introduction}

An eating disorder (ED) is a disturb characterized by an atypical eating behavior. Studies show that at least 70 million of people in the planet are affected by this type of disturb [Renfrew Center Foundation For Eating Disorders 2003]. A person with an eating disorder usually experiences extreme conditions such as severe overeating or reduction of food intake, often accompanied by feelings of concern about body image and physical appearance [National Institute of Mental Health 2007]. According to social psychology, those feelings usually arise from the contact with distorted beliefs when facing triggering situations, which may lead to an increase in anxiety levels, causing abnormal behaviors.

The most commonly adopted strategy to treat patients suffering from an eating disorder is cognitive-behavioral therapy (CBT) [Schiraldi 2016], which aims at modifying possible distorted beliefs regarding the disorder. However, the conventional approaches i.e., through patients memory and imagination or real situations (in vivo exposure), have some limitations. In this scenario, virtual reality (VR) has been shown useful to simulate the real world, providing an alternative to evoke thoughts about the subject's own feelings and behaviors.

In fact, virtual environments have been widely used for clinical treatment of individuals with psychological disorders, in neuropsychology and anxiety disorders 
such as agoraphobia [North et al. 1996], acrophobia [Opdyke et al. 1995], spider phobia [Carlin et al. 1997], fear of public speaking (social phobia) [North et al. 2015], claustrophobia [Botella et al. 1998], and flying phobia [North and North 1994]. In eating disorders, virtual reality has been used mainly to address body image disturbance [Riva and Melis 1997], often overlooked in ED treatments, and relationship with food through exposure therapy [Cardi et al. 2012].

In addition to VR approaches, another successful strategy for treating psychological disorders is the use of serious games, which have been shown of promising potential in health context due to its advantages when compared to other approaches [Pereira et al. 2014]. Serious games are an additional form of treatment in several areas, for enhancing positive attitudes [Beale et al. 2007, Rassin et al. 2004], increasing problem solving strategies [Coyle et al. 2005] and modifying some abnormal behaviors [Walshe et al. 2003].

Thus, in this work we propose a new approach for enhancing eating disorders treatment, using virtual reality and concepts of serious games and gamification. Our main goal is to create and validate an immersive virtual environment where the existent therapy approaches are experienced as a game, reflecting part of the patient's daily routine. This environment is intended to be the starting point for a complete treatment system, which will be thereafter designed to identify disturbances in anxiety levels based on the behavior of the patient during the game, alerting the therapist to possible anxiety triggers that may lead to behaviors associated to the eating disorder.

The role of VR here is to support the flexibilization of beliefs. The idea is to work with controlled risk exposure by checking anxiety levels. For example, if anxiety increases, compulsion aggravates, if anxiety decreases, the compulsive behavior, whether it is to overeat or undereat, is activated from a cognitive evaluation that prefers to maintain the attitude historically understood as correct by the person with the eating disorder. We also want to further investigate whether it is easier for the individual to identify and modify distorted beliefs if they are associated with everyday activities, i.e. watching television or talking to friends.

\section{Background and Related Work}

In social cognition, a field of social psychology, disturbs in behavior can be consequence of cognitive distortions, that is, inaccurate and constant patterns of thinking leading to varying degrees of distortion of reality [Beck 1963]. These distortions come from dysfunctional beliefs based on an individual's perception about himself or herself, mainly formed by comparison with other people, that reflect how they observe themselves and how they would like to be, the "real me" and "ideal me", respectively [Rodrigues et al. 2000]. In eating disorders context, such distortions can be understood as "a biased way of thinking about oneself or one's environment, including one's body image, weight or appearance" [Shepphird 2009].

Eating disorders are commonly classified in anorexia nervosa (AN), bulimia nervosa $(\mathrm{BN})$, Binge Eating disorder (BED) and Eating Disorder not Otherwise Specified (EDNOS). Besides the differences among them and the implications each one brings to the patients' health, they all share common principles and symptoms. Anxiety and depression are some of those principles, both are strongly linked to eating disorders and 
often reinforce disturbed patterns that, when fed, raise anxiety and depression levels even further, causing the patient to be stuck in a cycle.

Binge eating, for example, is a socially learned disturb, and a person's attitude to regulate their body image implies an increase in anticipatory anxiety in two ways. On one hand, compulsion leads to consumption of an inadequate amount of food, which has a negative impact on the interpretation of a person's body image, raising anxiety. On the other hand, regulating food within reasonable limits implies making contact with the belief of inadequacy underlying body image, which also raises anxiety.

Thus, anxiety is the prevailing emotion, and the strategy adopted, whether of avoidance or control, feeds the system that is governed by the person's attitude. Attitude is a deep-rooted belief that, according to [Baumeister and Finkel 2010], is consistent with the displayed behavior. That is, the attitude predicts the behavior which, in turn, corroborates the attitude. Hence, the rigidity of attitude that sustains dysfunctional eating behavior is the target. If we want to change behavior, we need to challenge this deeprooted belief and the cognitive distortions associated with it.

In this sense, CBT understands that emotions and behaviors are generated in function of thoughts which, in turn, are build upon the beliefs and schemes of each individual. Based on this principle, CBT techniques seek to eliminate and replace cognitive distortions, often including exposure to situations in which the patient is encouraged to think about his or her attitudes. Besides modifying the distorted beliefs associated with weight, appearance and eating habits, CBT usually seeks to increase adherence to treatment, increase or decrease weight (when applicable), develop a regular and flexible eating pattern, reduct body image disturbance, develop interpersonal abilities, increase self-esteem and prevent relapses [Cordioli 2009].

As already mentioned, VR has been widely used as an alternative to overcome memory and imagination drawbacks that can occur on CBT. There are many reasons for that. VR exposure makes it possible to explore multiple scenarios and different difficulty levels, increasing motivation and reducing the recurrence of fear and return of fear post treatment [Maldonado et al. 2017], since patients feel challenged and efficacious once they overcome feared situations [Riva and Mantovani 2012]. The sense of presence is another advantage of VR-based systems, given that is it possible to provide credible experiences demonstrating that distorted assumptions are not actually true [Riva et al. 2004].

Another definition for VR in this context is an "embodied technology", that is, the possibility of the subjects to be present in the virtual environment not through their physical bodies, but instead, through the modification of cognitive factors that regulate our experience of body and space, in such way that the physical body is somewhat replaced by the virtual avatar [Riva et al. 2015]. Additionally, because therapists can maintain a strict control over the variables assessed and, consequently, over the anxiety levels to which the patient is exposed, and since the patient does not have to really face the feared situation, VR offers a higher level of control and a more secure environment for the patient than that offered by direct "real" experience [North et al. 1997], minimizing chances of anxiety or panic attacks.

Most of the research regarding virtual reality applications to eating disorders has been conducted by two groups: Riva's group in Italy (54\%) and the group led by Perpiñá, 
Botella, and Baños in Spain (42\%) [Clus et al. 2018]. Riva et al. were the first to design and evaluate a set of virtual scenarios, called The Virtual Environment for Body Image Modification (VEBIM). In these scenarios, users have specific tasks such as weighting themselves, discussing feelings and beliefs elicited by a room with pictures of models, choosing bodies considered ideal and real, etc. Perpiñá also created an environment made of scenarios, rooms with mirrors, pictures of people with different shapes and characteristics, virtual food, modeling of desired and subject bodies using a 3D avatar, and so on.

Such existing strategies usually focus on recreating isolated tasks already present in CBT, by exposing the patient to food and different body types, replacing traditional image exposure, or reproducing scenarios that possibly trigger dysfunctional beliefs, avoiding imagination problems. They often do not consider activities as part of the patient's day. Also, no mechanisms for measuring anxiety levels or predicting anxiety disturbances based on the patient's decisions within the environment were identified in the existing systems, which could be of great help to the therapist to identify, analyze and discuss possible distorted cognitions during the game.

Besides VR strategies, [Fernández-Aranda et al. 2012] developed PlayMancer, a serious game designed for treating specific mental disorders (EDs and impulse control disorders). Preliminary results showed that, after using PlayMancer, patients started to show new coping styles with negative emotions in normal stress life situations, additional generalization patterns and more self-control strategies. [Walshe et al. 2003] investigated the effectiveness of the combined use of VR and serious games in exposure therapy for fear of driving. The findings of this study suggest that virtual reality and game reality (GR) together may have a useful role in the treatment of driving phobia post accident even when co-morbid conditions such as post-traumatic stress disorder and depression co-exist, though findings would suggest that inducing presence/immersion in a greater percentage of subjects would increase the viability of VR/GR as an exposure treatment for driving anxiety.

\section{Proposed Environment}

The proposed environment was developed using Unity, a multi-platform game engine with a free version that offers good graphic quality, enhanced using the existent post-processing tool, free built-in functions and scripts, and runs with average PC requirements, and Oculus Rift, a virtual reality immersive system with low cost and high quality, alongside with Oculus touch controllers.

\subsection{Game Script}

The game script was written focusing on reflecting a normal day in the patient's life. Activities were designed following CBT therapist guidance, resulting in exposition to different body types and shapes, food planning, virtual food intake, and other possible triggering events, inserted in everyday activities such as watching television, talking to friends, planning meals and even looking at the mirror. That way, the patient learns to identify and modify dysfunctional beliefs when similar events occur even outside VR environment, not limiting treatment to clinic.

The game scenes were designed to reflect parts of the day focusing on the most common cognitive distortions when talking about eating disorders: food intake, social 
skills and body image. For food intake, three scenes with basic meals were developed: breakfast, lunch and dinner. Figure 1 shows those scenes. The proposed meal plan was designed by a nutritional counselor, and every ingredient was chosen in order to fulfill minimum daily nutrition requirements. In each scene, the patient can choose between eating or not, as well as ingredients and portions. Additionally, a list with food recommendations for that specific meal is available. It is possible to see the list by looking down, as shown in Figures $1 \mathrm{~b}$ and 1d. Note that there are two language options for the list, as well as for the whole game, English and Portuguese.

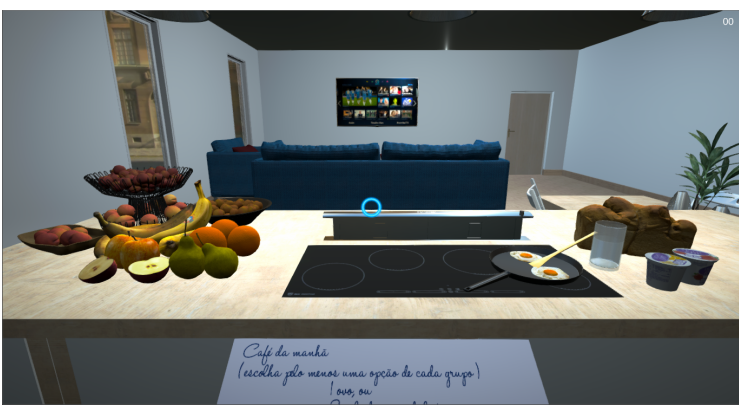

(a) Breakfast scene

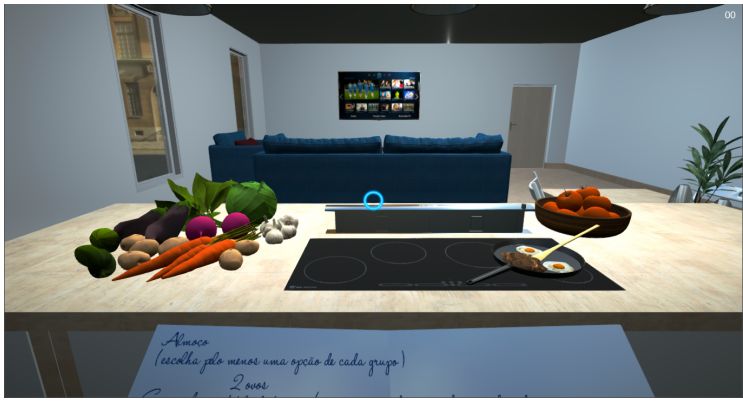

(c) Lunch/Dinner scene

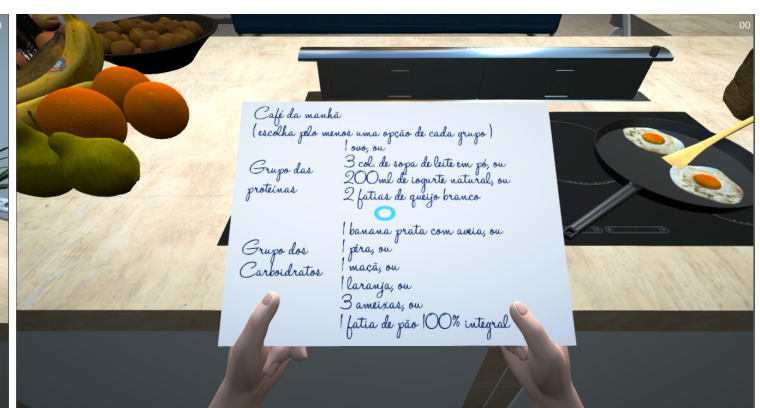

(b) Breakfast list

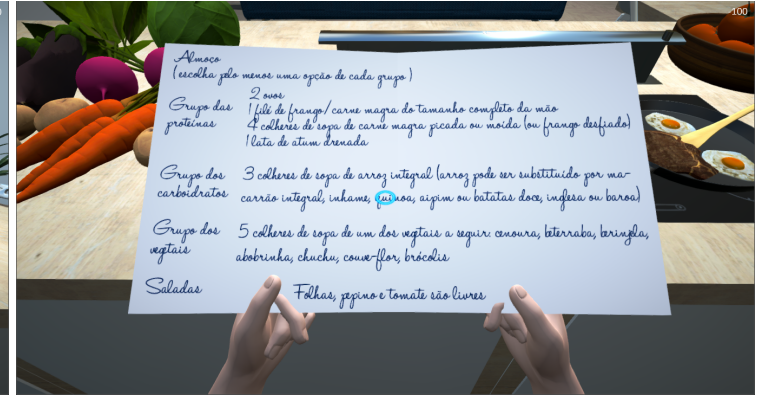

(d) Lunch/Dinner list

Figure 1. Food intake scenes: breakfast, lunch and dinner. (1a) and (1c) show the food choice environment for breakfast (1a) and lunch or dinner (1c). (1b) and (1d) show the list with food recommendations for each meal.

Ingredients are highlighted when the patient looks at it, and it is possible to select it by pressing a button on touch controller. When a correct ingredient is chosen, i.e, it is present in nutritional recommendations, it automatically becomes checked on the list, so the patient knows his/her choice fulfills the minimum nutrition requirements. After choosing all the desired ingredients, the patient can confirm or change his/her decisions. Decisions according to the nutrition plan, that is, that modify distorted beliefs, increase the score, while decisions that do not change beliefs related to eating disorder behaviors decrease the score.

In the social skills scene, a friend sends a message asking if it is possible to have lunch at the patient's house. This happens between breakfast and lunch. Again, the patient can chose to accept or make an excuse, and the score changes according to his/her behavior. Note that the decision to have lunch or not, mentioned above, does not depends on this one. It is possible to let the friend come and yet do not have lunch, but this action will affect the dialogue between the patient and his/her friend. 
For body image, two scenes were developed. They are shown in Figure 2. In the first one, the patient is watching TV and two videos are displayed. One is an advertisement showing Victoria Secret's models, and the other is a fashion show from Addition Elle, with plus size models. The patient is then encouraged to think about his/her feelings and actions towards such media exposure. This happens before lunch. In the second scene, the patient is looking at his/her body in a "smart mirror", and it is possible to adjust the size of the arms, chest, waist, hips and legs. If choices do not match the patient's measurements, the score is decreased and the patient can chose to come back and change the avatar or ignore it (in both choices, real body measurements are contrasted to the patient's modeled 3D avatar). Figure $2 \mathrm{~b}$ shows three possibilities for adjustments made by the patient, representing normal weight, overweight and underweight, respectively.

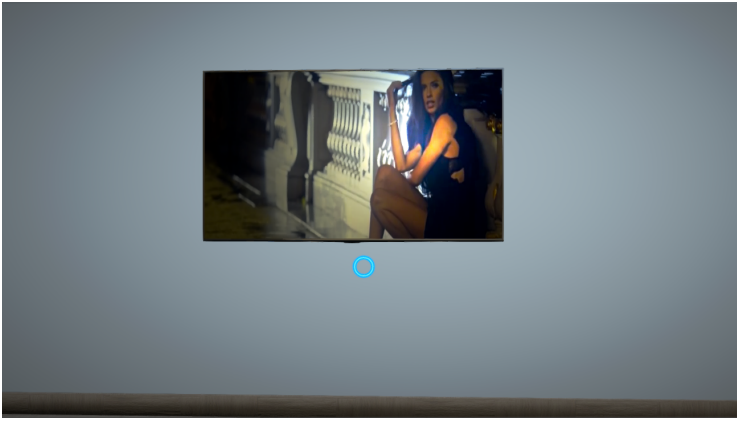

(a) Body image scene: TV
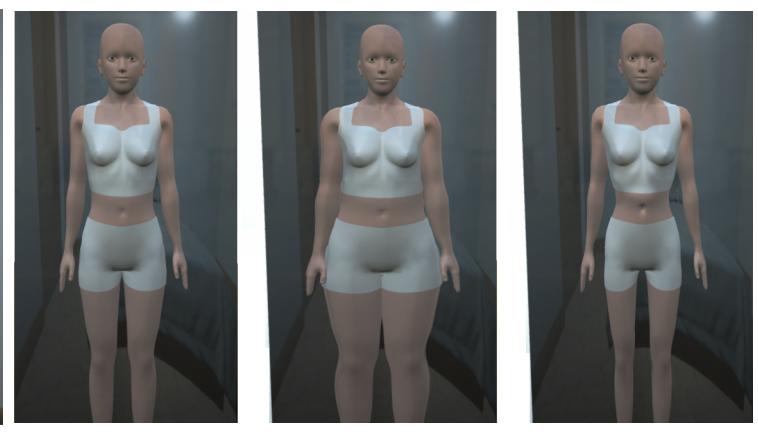

(b) Body image scene: smart mirror

Figure 2. (2a) and (2b) show body image scenes. In (2a), the patient is watching to a Victoria's Secrets advertisement, while in (2b), patient can adjust his/her body reflected in a smart mirror.

As in real life, the patient has total control over his/her decisions throughout the game. However, the therapist supervises the game walkthrough in real time and may encourage the patient to think about reasons and feelings behind each decision. In addition, decisions that lower the patient's score affects the energy the patient has to keep playing, and have consequences on next scenes.

\section{System Evaluation and Discussion}

Two tests were performed in order to validate the virtual environment. The first one, SUS, verifies the usability of the system, while the second one, IPQ, measures the user's sense of presence in the virtual environment. Tests were applied to a group of ten volunteer participants, six men and four women aged between 23 and 30. All of the participants declared that they a) do not suffer from an ED, b) frequently use computers and other electronic systems, and c) usually play some entertainment game on a daily basis or several times a week. When questioned about their experience with VR environments, five of them declared to have already used head mounted displays and controllers at least once.

\subsection{SUS Test}

The System Usability Scale is one of the simplest and most used methods for measuring usability, i.e, the ease of use of systems. This tool helps to evaluate system effectiveness and efficiency, as well as user satisfaction. We use the SUS because usability is a fundamental factor for the success of our system, since the patient should feel comfortable and 
be able to quickly master the system's functionalities, therefore increasing adherence to treatment. SUS consists of ten questions, present in Table 1, that can be answered on a five-point Likert scale of "Strongly Disagree" to "Strongly Agree".

Table 1. Questions used on System Usability Scale (SUS)

\begin{tabular}{|c|l|}
\hline 1 & I think that I would like to use this system frequently. \\
\hline 2 & I found the system unnecessarily complex. \\
\hline 3 & I thought the system was easy to use. \\
\hline 4 & I think that I would need assistance to be able to use this system. \\
\hline 5 & I found the various functions in this system were well integrated. \\
\hline 6 & I thought there was too much inconsistency in this system. \\
\hline 7 & I would imagine that most people would learn to use this system very quickly. \\
\hline 8 & I found the system very cumbersome/awkward to use. \\
\hline 9 & I felt very confident using the system. \\
\hline 10 & I needed to learn a lot of things before I could get going with this system. \\
\hline
\end{tabular}

Individual scores for each of the ten participants are shown in Table 2. The System Usability Scale score, obtained from answers of the ten participants, was 78.3 in a range from 0 to 100. Studies show that a SUS score of 68 is the average score, and anything below that suggests usability issues. The standard deviation for this score was 10.3, with a coefficient of variation equals to $13 \%$, indicating an homogeneous sample, which means that scores are mainly close to the average. Additionally, by computing the margin of error, we found out that the true mean of the population, that is, the real score, is inside the interval from 71.92 to 84.68 with a $95 \%$ confidence level.

Table 2. Participants individual scores for SUS

\begin{tabular}{|c|c|}
\hline Participant & Score \\
\hline $\mathrm{p} 1$ & 75.0 \\
\hline $\mathrm{p} 2$ & 67.5 \\
\hline $\mathrm{p} 3$ & 87.5 \\
\hline $\mathrm{p} 4$ & 72.5 \\
\hline $\mathrm{p} 5$ & 65.0 \\
\hline $\mathrm{p} 6$ & 90.0 \\
\hline $\mathrm{p} 7$ & 87.5 \\
\hline $\mathrm{p} 8$ & 72.5 \\
\hline $\mathrm{p} 9$ & 95.0 \\
\hline $\mathrm{p} 10$ & 77.5 \\
\hline
\end{tabular}

\subsection{IPQ Test}

The sense of presence is a crucial factor in VR systems for the treatment of eating disorders, because it helps to provide credible experiences demonstrating that distorted assumptions are not actually true [Riva et al. 2004], specially about body image. It is important to notice that sense of presence is not the same as immersion, because sense of presence depends on a user's experience rather than on the technology used. 
The Igroup Presence Questionnaire (IPQ) is a scale for measuring the sense of presence experienced in a virtual environment. This test comprises fourteen items directly availed (the IPQ questions) rated on a seven-point Likert Scale, from 0 to 6, where each question is related to a specific subscale that can be studied indirectly. The General item group assesses the general sense of "being there", and has high loadings on all three following factors. The Spatial Presence (SP) subscale is related to the sense of being physically inside the environment. The Involvement (INV) subscale evaluates the attention devoted to the environment. Last, the Realness (REAL) subscale measures the sense of reality attributed to the environment.

The mean scores for each question are shown in Table 3, and the mean scores for the subscales are shown in Table 4. From Table 3, we can see that all questions had a score above average, i.e, above 3 . In its turn, Table 4 indicates that individuals had a great sense of being physically in the environment, due to the Spatial Presence score, of 5.34. The involvement subscale received a score of 4.8, and the Realness subscale scored 4.35, showing also satisfactory levels for the involvement experienced and the experience of realism. Table 4 also presents standard deviations and confidence interval values. From this data, we conclude, with $95 \%$ of confidence level, that the true mean score for all subscales lays between 4.05 and 5.13, indicating that the environment offers a great sense of presence, crucial for eating disorders treatment applications.

Table 3. Mean scores for the fourteen questions used on IPQ

\begin{tabular}{|c|c|c|c|}
\hline 1 & sense of being there & G & 5.4 \\
\hline 2 & sense of virtual environment behind & SP & 5.6 \\
\hline 3 & feeling like just perceiving pictures & SP & 4.9 \\
\hline 4 & not sense of being in virtual space & SP & 5.5 \\
\hline 5 & sense of acting in VE & SP & 5.1 \\
\hline 6 & sense of being present in VE & SP & 5.6 \\
\hline 7 & awareness of real environment & INV & 3.9 \\
\hline 8 & not aware of real environment & INV & 5.4 \\
\hline 9 & no attention to real environment & INV & 4.2 \\
\hline 10 & attention captivated by VE & INV & 5.7 \\
\hline 11 & VE real (real/not real) & REAL & 4.7 \\
\hline 12 & experience similar to real environment & REAL & 5.2 \\
\hline 13 & VE real (imagined/real) & REAL & 4.5 \\
\hline 14 & VE wirklich & REAL & 3.1 \\
\hline
\end{tabular}

Table 4. Mean scores, standard deviations (STD) and confidence intervals (CI) for IPQ subscales

\begin{tabular}{|c|c|c|c|}
\hline & Mean Score & STD & CI (95\%) \\
\hline General & 4.91 & 0.4 & $4.91 \pm 0.22$ \\
\hline Spatial Presence & 5.34 & 0.3 & $5.34 \pm 0.16$ \\
\hline Involvement & 4.8 & 0.4 & $4.8 \pm 0.24$ \\
\hline Realness & 4.35 & 0.5 & $4.35 \pm 0.31$ \\
\hline
\end{tabular}




\section{Conclusions and Future Directions}

Virtual reality environments and serious games have been widely used for the assessment and treatment of mental disorders. In the eating disorders context, existing VR applications focused on reproducing exposure therapy tasks, with no use of serious games or gamification concepts. On the other hand, VR has been used combined with serious games for treating another mental disorders, and results were promising. Additionally, no mechanisms for measuring disturbances in anxiety levels based on the patient's decisions were found in the existent environments.

Therefore, this work presented a new approach for enhancing eating disorders treatment, combining VR and serious games concepts. The game script was written focusing on reflecting a normal day in the patient's life, and activities were designed following a CBT therapist guidance. Six scenes were created, and the decisions made by the patient affects the game workflow. Also, the therapist watches the whole game walkthrough, discussing reasons and feelings that may have originated patient's decisions. Scenes regarding food were planned with nutritionist counseling.

Two tests were performed in order to validate the virtual environment. SUS and IPQ. An statistic analysis of SUS score indicated that the environment usability is above average, giving a score of 78.3 (average is 68), which was considered satisfactory. IPQ scores also revealed a great sense of presence induced by the virtual environment, with spatial presence being the highest score (5.34) in a scale from 0 to 6 , which is a crucial factor for identifying and replacing cognitive distortions.

From those results we conclude that our virtual environment is easy to use and provides a great sense of presence, using lower-cost equipment and following therapist and nutritional guidance. Future work concerns an analysis of the anxiety levels generated by the virtual environment, as well as case studies, non-controlled and controlled clinical trials, in order to investigate the effectiveness of the system for treating eating disorder disturbs.

Also, as already mentioned, the environment created was intended to be the starting point for a complete treatment system that will focus on identifying disturbances in anxiety levels, so that the therapist can detect exactly which experience elicited distorted behaviors and work with the patient on that. Last, to provide an even more controlled exposure and avoid traumas, we intend to create levels for each scene, making it possible for the therapist to control the level of anxiety the patient can be exposed to, based on the patient's history and on treatment evolution.

\section{References}

Baumeister, R. F. and Finkel, E. J. (2010). Advanced social psychology: The state of the science. OUP USA.

Beale, I. L., Kato, P. M., Marin-Bowling, V. M., Guthrie, N., and Cole, S. W. (2007). Improvement in cancer-related knowledge following use of a psychoeducational video game for adolescents and young adults with cancer. Journal of Adolescent Health, 41(3):263-270.

Beck, A. T. (1963). Thinking and depression: I. idiosyncratic content and cognitive distortions. Archives of general psychiatry, 9(4):324-333. 
Botella, C., Baños, R., Perpiñá, C., Villa, H., Alcaniz, M., and Rey, A. (1998). Virtual reality treatment of claustrophobia: a case report. Behaviour research and therapy, 36(2):239-246.

Cardi, V., Krug, I., Perpiñá, C., Mataix-Cols, D., Roncero, M., and Treasure, J. (2012). The use of a nonimmersive virtual reality programme in anorexia nervosa: A single case-report. European Eating Disorders Review, 20(3):240-245.

Carlin, A. S., Hoffman, H. G., and Weghorst, S. (1997). Virtual reality and tactile augmentation in the treatment of spider phobia: a case report. Behaviour research and therapy, 35(2):153-158.

Clus, D., Larsen, M. E., Lemey, C., and Berrouiguet, S. (2018). The use of virtual reality in patients with eating disorders: Systematic review. Journal of medical Internet research, 20(4).

Cordioli, A. V. (2009). Psicoterapias: Abordagens atuais. Artmed Editora.

Coyle, D., Matthews, M., Sharry, J., Nisbet, A., and Doherty, G. (2005). Personal investigator: A therapeutic 3d game for adolecscent psychotherapy. Interactive technology and smart education, 2(2):73-88.

Fernández-Aranda, F., Jiménez-Murcia, S., Santamaría, J. J., Gunnard, K., Soto, A., Kalapanidas, E., Bults, R. G., Davarakis, C., Ganchev, T., Granero, R., et al. (2012). Video games as a complementary therapy tool in mental disorders: Playmancer, a european multicentre study. Journal of Mental Health, 21(4):364-374.

Maldonado, J. G., Ferrer García, M., Dakanalis, A., and Riva, G. (2017). Virtual reality: applications to eating disorders.

National Institute of Mental Health (2007). Eating disorders.

North, M. and North, S. (1994). Virtual environments and psychological disorders. Electronic Journal of Virtual Culture, 2(4):37-42.

North, M. M., North, S. M., and Coble, J. R. (1996). Effectiveness of virtual environment desensitization in the treatment of agoraphobia. Presence: Teleoperators \& Virtual Environments, 5(3):346-352.

North, M. M., North, S. M., and Coble, J. R. (1997). Virtual reality therapy: An effective treatment for psychological. Virtual reality in neuro-psycho-physiology: Cognitive, clinical and methodological issues in assessment and rehabilitation, 44:59.

North, M. M., North, S. M., and Coble, J. R. (2015). Virtual reality therapy: an effective treatment for the fear of public speaking. International Journal of Virtual Reality (IJVR), 3(3):1-6.

Opdyke, D., Williford, J. S., and North, M. (1995). Effectiveness of computer-generated (virtual reality) graded exposure in the treatment of acrophobia. Am J psychiatry, 1(152):626-28.

Pereira, P., Duarte, E., Rebelo, F., and Noriega, P. (2014). A review of gamification for health-related contexts. In International conference of design, user experience, and usability, pages 742-753. Springer. 
Rassin, M., Gutman, Y., and Silner, D. (2004). Developing a computer game to prepare children for surgery. AORN journal, 80(6):1095-1102.

Renfrew Center Foundation For Eating Disorders (2003). Eating disorders 101 guide: A summary of issues, statistics and resources.

Riva, G., Dakanalis, A., and Mantovani, F. (2015). Leveraging psychology of virtual body for health and wellness. The Handbook of the Psychology of Communication Technology. Chichester, UK: John Wiley \& Sons, Ltd, pages 528-47.

Riva, G. and Mantovani, F. (2012). Being there: Understanding the feeling of presence in a synthetic environment and its potential for clinical change. In Virtual reality in psychological, medical and pedagogical applications. InTech.

Riva, G., Mantovani, F., and Gaggioli, A. (2004). Presence and rehabilitation: toward second-generation virtual reality applications in neuropsychology. Journal of neuroengineering and rehabilitation, 1(1):9.

Riva, G. and Melis, L. (1997). Virtual reality for the treatment of body image disturbances. Studies in health technology and informatics, pages 95-112.

Rodrigues, A., Assmar, E. M. L., and Jablonski, B. (2000). Psicologia social (ref.). Petrópolis: Vozes.

Schiraldi, G. R. (2016). The self-esteem workbook. New Harbinger Publications.

Shepphird, S. F. (2009). 100 questions \& answers about anorexia nervosa. Jones \& Bartlett Publishers.

Walshe, D. G., Lewis, E. J., Kim, S. I., O'Sullivan, K., and Wiederhold, B. K. (2003). Exploring the use of computer games and virtual reality in exposure therapy for fear of driving following a motor vehicle accident. CyberPsychology \& Behavior, 6(3):329334. 